\title{
La Dieta y la Salud del Cerebro'
}

\author{
Pooja Tolani and Wendy J. Dahl²
}

Seguir una dieta saludable proporciona muchos beneficios a lo largo del ciclo de la vida. Mantener buenos hábitos de alimentación a medida que envejecemos puede ser especialmente útil para preservar la salud cognitiva-la capacidad de recordar, aprender y tomar decisiones. Ésta publicación proporciona consejos para una alimentación saludable que puede ayudar a maximizar la salud del cerebro en adultos mayores.

\section{¿Qué alimentos debo comer para apoyar la salud del cerebro?}

Los patrones dietéticos conocidos por promover la salud del corazón también pueden ser beneficiosos para la salud del cerebro. El patrón dietético Mediterráneo hace énfasis en los cereales integrales, las legumbres, las verduras, las frutas, las nueces, el aceite de oliva y el pescado, y adicionalmente cantidades moderadas de carne y productos lácteos (Widmer et al. 2015). Este patrón dietético también incluye el vino, aunque no se recomienda comenzar a consumir alcohol si no lo ha hecho anteriormente. Seguir un patrón dietético mediterráneo se ha relacionado con un menor riesgo de deterioro cognitivo, enfermedad de Alzheimer y otras formas de demencia (van de Rest et al. 2015) y puede mejorar la función cognitiva en adultos mayores (VallsPedret et al. 2015).

Otro patrón dietético que puede ser beneficioso para la salud del cerebro es la dieta DASH, por sus siglas en inglés (Solfrizzi et al. 2017), una dieta que se recomienda para disminuir la presión arterial y el riesgo de enfermedad cardíaca (Siervo et al. 2015). La dieta DASH se enfoca en frutas, vegetales, productos lácteos bajos en grasa, granos enteros, pollo, pescado y nueces. Se les recomienda a las personas que siguen este patrón dietético que limiten su consumo de carnes rojas, dulces, bebidas que contengan azúcar y alimentos con alto contenido de grasas saturadas.

Como los alimentos recomendados en la dieta mediterránea y la dieta DASH se han encontrado útiles para mantener la salud del cerebro, la combinación de los alimentos recomendados en cada uno de éstos patrones dietéticos puede ser aún más beneficioso. La dieta MIND, por sus siglas en inglés ha sido probada por su eficacia para proteger la salud del cerebro. Esta dieta se centra en más alimentos a base de plantas y menos alimentos de origen animal (Marcason 2015). La dieta MIND ha sido relacionada con un menor riesgo de desarrollar la enfermedad de Alzheimer (Morris et al. 2015).

La Tabla 1 muestra los alimentos que pueden ayudar a proteger la salud del cerebro (Marcason 2015). Procure consumir al menos 5 porciones de verduras al día, especialmente de hojas verdes (por ejemplo, espinaca, col, y col rizada) y vegetales crucíferos (por ejemplo, brócoli, repollo, y coles de Bruselas). Consuma al menos 4 porciones de frutas cada día, eligiendo bayas (como por ejemplo fresas, arándanos) con frecuencia. Los ácidos grasos Omega-3, que se encuentran en alimentos como el aceite de oliva, las nueces y los pescados grasos (por ejemplo, el salmón, el atún y las sardinas) ayudan a mantener la salud del cerebro. Elija nueces sin sal o mantequilla de nueces casi todos

1. Este documento, FSHN18-4, es uno de una serie de publicaciones del Food Science and Human Nutrition, UF/IFAS Extensión. Fecha de primera

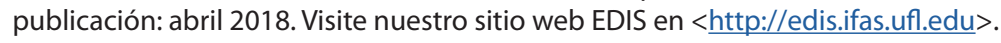

2. Pooja Tolani, dietetic intern; and Wendy J. Dahl, associate professor; Food Science and Human Nutrition Department, UF/IFAS Extension, Gainesville, FL 32611.

The Institute of Food and Agricultural Sciences (IFAS) is an Equal Opportunity Institution authorized to provide research, educational information and other services

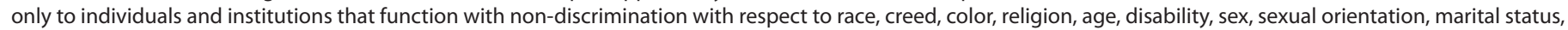

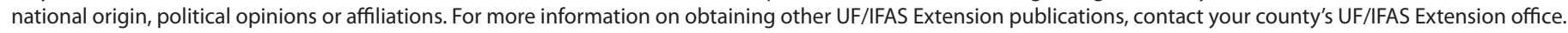
U.S. Department of Agriculture, UF/IFAS Extension Service, University of Florida, IFAS, Florida A \& M University Cooperative Extension Program, and Boards of County Commissioners Cooperating. Nick T. Place, dean for UF/IFAS Extension. 
los días. Se recomienda una comida basada en legumbres (por ejemplo, garbanzos, lentejas, y variedad de frijoles) al menos dos veces a la semana. Elija pescado al menos 3 veces por semana.

\section{¿Qué alimentos debo limitar para apoyar la salud del cerebro?}

El patrón dietético MIND enfatiza comer más alimentos a base de plantas y limitar los alimentos de origen animal con alto contenido de grasas saturadas. Las grasas saturadas son sólidas a temperatura ambiente y se encuentran en productos de origen animal como la mantequilla, la crema y la grasa dentro y alrededor de la carne, así como en la margarina, la panadería y los refrigerios hechos con aceites vegetales hidrogenados. Para promover la salud del cerebro, evite los alimentos horneados comprados en las tiendas, como rosquillas, pasteles y galletas, así como refrigerios azucarados y salados y la mayoría de las comidas pre empaquetadas, congeladas y enlatadas. En La Guía de Alimentos Saludables Para el Cerebro, se recomienda limitar la carne y las aves a no más de una comida por día, y las carnes rojas y procesadas a menos de una vez a la semana. La Tabla 2 enumera alimentos que deben ser limitados o evitados para proteger la salud del cerebro (Marcason 2015).

\section{¿Debería de preocuparme por ciertos nutrientes específicos?}

A medida que envejecemos, hay un mayor riesgo de deficiencia de vitamina B12. Como la vitamina B12 ayuda a mantener el cerebro y el sistema nervioso saludables, es importante consumir alimentos de origen animal que contengan vitamina $\mathrm{B} 12$, como productos lácteos, carne, pescado, aves de corral y huevos. Para aquellos que consumen solo alimentos de origen vegetal, se recomienda elegir alimentos fortificados con vitamina B12 o tomar un suplemento de vitamina B12. Algunos ejemplos de alimentos que pueden ser enriquecidos con vitamina B12 incluyen leches no lácteas (por ejemplo, leche de soya), sustitutos de la carne (por ejemplo, tofu) y ciertos cereales para el desayuno.

Los adultos mayores que consumen cantidades adecuadas de verduras, frutas, cereales integrales, pescado y productos lácteos bajos en grasa tienden a tener un mejor estado nutricional y calidad de vida en general (Bernstein and Muñoz 2012). De manera similar, la dieta MIND enfatiza estos grupos de alimentos importantes y puede ayudar a retrasar o prevenir la alteración de la memoria y aquellas enfermedades relacionadas con el cerebro, como la enfermedad de Alzheimer (Morris et al. 2015). Incluir una amplia variedad de alimentos de los patrones dietéticos Mediterráneo, DASH y MIND puede ser beneficioso para la salud del cerebro, y también proporcionará los nutrientes necesarios para mantener una buena salud y mejor calidad de vida.

\section{References}

Bernstein, M., and N. Munoz. 2012. "Position of the Academy of Nutrition and Dietetics: food and nutrition for older adults: promoting health and wellness." J Acad Nutr Diet 112(8):1255-77. doi:10.1016/j.jand.2012.06.015.

Marcason, W. 2015. "What Are the Components to the MIND Diet?" J Acad Nutr Diet 115(10):1744. doi:10.1016/j. jand.2015.08.002.

Morris, M.C., C.C. Tangney, Y. Wang, F.M. Sacks, D.A. Bennett, and N.T. Aggarwal. 2015. "MIND diet associated with reduced incidence of Alzheimer's disease." Alzheimers Dement 11(9):1007-14. doi:10.1016/j.jalz.2014.11.009.

Siervo, M., J. Lara, S. Chowdhury, A. Ashor, C. Oggioni, and J.C. Mathers. 2015. "Effects of the Dietary Approach to Stop Hypertension (DASH) diet on cardiovascular risk factors: a systematic review and metaanalysis." Br J Nutr 113(1):1-15. doi: 10.1017/s0007114514003341.

Solfrizzi, V., C. Custodero, M. Lozupone, B.P. Imbimbo, V. Valiani, P. Agosti, A. Schilardi, A. D’Introno, M. La Montagna, M. Calvani, V. Guerra, R. Sardone, D. I. Abbrescia, A. Bellomo, A. Greco, A. Daniele, D. Seripa, G. Logroscino, C. Sabba, and F. Panza. 2017. "Relationships of Dietary Patterns, Foods, and Microand Macronutrients with Alzheimer's Disease and Late-Life Cognitive Disorders: A Systematic Review." J Alzheimers Dis 59(3):815-849. doi: 10.3233/jad-170248.

Valls-Pedret, C., A. Sala-Vila, M. Serra-Mir, D. Corella, R. de la Torre, M.A. Martinez-Gonzalez, E.H. MartinezLapiscina, M. Fito, A. Perez-Heras, J. Salas-Salvado, R. Estruch, and E. Ros. 2015. "Mediterranean Diet and Age-Related Cognitive Decline: A Randomized Clinical Trial." JAMA Intern Med 175(7):1094-103. doi: 10.1001/ jamainternmed.2015.1668.

van de Rest, O., A.A. Berendsen, A. Haveman-Nies, and L.C. de Groot. 2015. "Dietary patterns, cognitive decline, and dementia: a systematic review." Adv Nutr 6(2):154-68. doi: 10.3945/an.114.007617. 
Widmer, R.J., A.J. Flammer, L.O. Lerman, and A. Lerman.

2015. "The Mediterranean diet, its components, and

cardiovascular disease." Am J Med 128(3):229-38. doi:

10.1016/j.amjmed.2014.10.014.

Table 1. Alimentos que aumentar para promover la salud del cerebro.

\begin{tabular}{|c|c|}
\hline Alimentos Saludables Para el Cerebro & Ejemplos \\
\hline Vegetales de hojas verdes & $\begin{array}{l}\text { Espinacas, col rizada, berza, acelgas, hojas de mostaza, hojas de nabo, hojas } \\
\text { de diente de león, rúcula, endivia, hojas de uva, lechuga romana }\end{array}$ \\
\hline Vegetales & $\begin{array}{l}\text { Espárragos, brócoli, coles de Bruselas, repollo, zanahorias, coliflor, berenjena, } \\
\text { cebolla, okra (quimbombó), guisantes de nieve, calabaza, pimientos, batatas, } \\
\text { tomates }\end{array}$ \\
\hline Nueces & Nueces, maní, almendras, marañón, pistachos o mantequilla de nueces \\
\hline Bayas & Arándanos, fresas, frambuesas, moras \\
\hline Legumbres & $\begin{array}{l}\text { Frijoles negros, pintos, cannellini, garbanzo, lima, rojo / blanco y azul marino; } \\
\text { lentejas, edamame }\end{array}$ \\
\hline Granos enteros & $\begin{array}{l}\text { Pan integral, arroz integral, pasta integral, arroz silvestre, quínoa, cebada, } \\
\text { búlgaro, avena, cereales integrales para el desayuno }\end{array}$ \\
\hline Pescado & Salmón, atún, tilapia, sardina, mahi mahi, pez hipogloso \\
\hline Aceite & Aceite de oliva extra virgen \\
\hline
\end{tabular}

Table 2. Alimentos que limitar para promover la salud del cerebro.

\begin{tabular}{|c|c|}
\hline Alimentos que limitar & Ejemplos \\
\hline Carne roja y carne procesada & $\begin{array}{l}\text { Carne de res, cordero, cerdo, jamón, hamburguesas, perros calientes, } \\
\text { salchichas, tocino, salami }\end{array}$ \\
\hline Grasas - sólidas a temperatura ambiente & Mantequilla, margarina en barra \\
\hline Queso & Quesos de leche entera \\
\hline Pasteles y dulces & $\begin{array}{l}\text { Bizcochos, pasteles, rollos dulces, pastelerías, donas, galletas, brownies, tarta } \\
\text { barras de caramelo, otros dulces, helados, bebidas azucaradas }\end{array}$ \\
\hline Alimentos fritos o rápidos & $\begin{array}{l}\text { Comida rápida o restaurantes rápidos casuales } \\
\text { Cualquier comida frita, incluidas las papas fritas }\end{array}$ \\
\hline
\end{tabular}

